\title{
Numerical exploration of vortex matter in Bose-Einstein condensates
}

\author{
L.O. Baksmaty ${ }^{\text {a }}$, Y. Liu ${ }^{\text {b }}$, U. Landman ${ }^{\text {c }}$, N.P. Bigelow ${ }^{\text {d }}$, H. Pu ${ }^{\mathrm{a}, *}$ \\ a Department of Physics and Astronomy, Rice University, Houston, TX 77251, United States \\ ${ }^{\mathrm{b}}$ School of Mathematics, Georgia Institute of Technology, Atlanta, GA 30332, United States \\ c School of Physics, Georgia Institute of Technology, Atlanta, GA 30332, United States \\ ${ }^{\mathrm{d}}$ Department of Physics and Astronomy, University of Rochester, Rochester, NY 14627, United States
}

Available online 18 June 2009

\begin{abstract}
We describe a finite element numerical approach to the full Hartree-Fock-Bogoliubov treatment of a vortex lattice in a rapidly rotating Bose-Einstein condensate. We study the system in the regime of high thermal or significant quantum fluctuations where we are presented with a very large nonlinear unsymmetric eigenvalue problem which is indefinite and which possesses low-lying excitations clustered arbitrarily close to zero, a problem that requires state-of-the-art numerical techniques.

(C) 2009 IMACS. Published by Elsevier B.V. All rights reserved.
\end{abstract}

PACS: $03.75 . \mathrm{Kk} ; 32.80 . \mathrm{Lg} ; 67.90 .+\mathrm{z} ; 67.40 . \mathrm{Vs}$

Keywords: Bose-Einstein condensate; Gross-Pitaevskii equation; Vortex; Finite element method

\section{Introduction}

As a hallmark of coherence in quantum many particle systems, quantized vortices are ubiquitous in superfluids and superconductors and the study of vortex physics is of both fundamental and practical importance $[14,8]$. The realization of trapped Bose-Einstein condensates (BECs) in dilute atomic vapors in 1995 offered a new platform for vortex studies (see Ref. [13] for a review). The BEC begins to nucleate in a fluid of bosonic particles below a transition temperature $\left(T_{c}\right)$ and is described by a macroscopic wavefunction $\Phi(\mathbf{r})=\rho_{c}(\mathbf{r}) e^{i S(\mathbf{r})}$, with an irrotational velocity: $\mathbf{v}_{c}(\mathbf{r})=(\hbar / m) \nabla S(\mathbf{r})$, where $m$ is the mass of the atom and $\hbar$ is Planck's constant. Upon rotation of the trap, the condensate is threaded by vortex lines which act as islands of normal fluid about which the condensed atoms can circulate. In this way the condensate is confined to a non-simply connected geometry and can acquire angular momentum without violating Stokes law. A vortex thus represents a singularity in the velocity field $\mathbf{v}_{c}$ and in the neighborhood of a vortex centered at $\mathbf{r}_{\mathbf{0}}, \nabla \times \mathbf{v}_{c}(\mathbf{r})=n \boldsymbol{\kappa} \delta\left(\mathbf{r}-\mathbf{r}_{0}\right)$, where $n$ is an integer and $\kappa=|\boldsymbol{\kappa}|=2 \pi \hbar / m$ is the quantum of vorticity.

The inhomogeneous vorticity of a BEC is in direct contrast to its uniformity in a solid body where, for a rotating frequency of $\Omega$, it has a value of $2 \Omega$. In equilibrium with a rotating container a BEC imitates a solid body by forming a vortex lattice (see Fig. 1) with an areal vortex density given by: $n_{v}=2 \Omega / \kappa$, which confers a coarse grained velocity

\footnotetext{
* Corresponding author.

E-mail address: hpu@ rice.edu (H. Pu).
} 

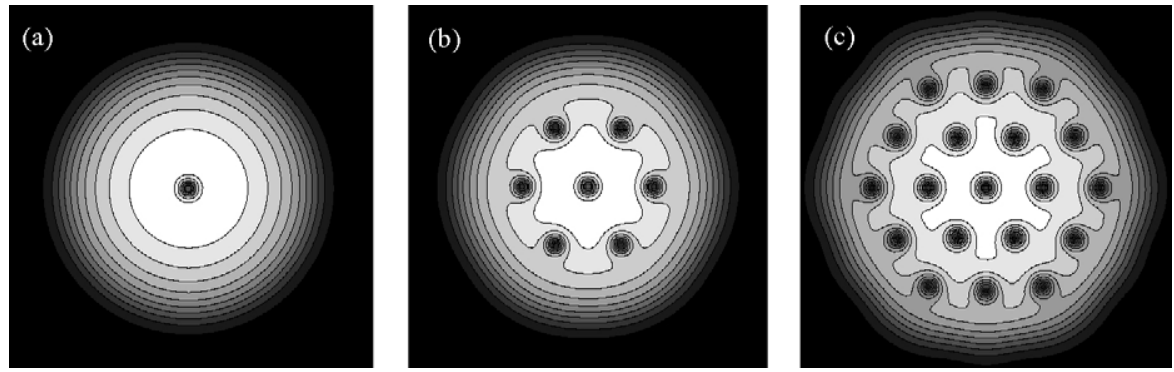

Fig. 1. Density contour plots of a rotating condensate. Lighter region represents higher density. From left to right the rotating frequency $\Omega$ of the BEC is increasing. As a consequence, the inter-vortex distance is decreasing, the radius of condensate grows and the number of vortices increases.

field of $\mathbf{v}_{c}^{a v}=\boldsymbol{\Omega} \times \mathbf{r}$ identical to a rigid body. This hydrodynamic picture of a condensate is however not the complete story.

Although there are theoretical speculations that as the filling fraction $v$ (the number of particles per vortex) approaches unity the vortex will increasingly behave like a quantum particle, and the system will form strongly correlated vortex liquid state analogous to the fractional quantum Hall systems [4], all experimental results to date are still within the mean-field regime that can be described using Hartree-Fock-Bogoliubov formalism. In this paper, we present the numerical techniques used in our recent studies of vortex matter in BEC based on this formalism.

\section{Physical Model}

Trapped rotating gases have acquired their special place because in addition to being free of impurity, the number of particles, the strength of inter-particle interaction, the angular velocity and the geometry of the trap and temperature, may be tuned over a very wide range. To be specific, we consider a system of $N$ bosonic atoms interacting via a contact potential and confined in an axi-symmetric harmonic potential rotating at frequency $\Omega$ about the symmetry axis $(z-$ axis): $V(\mathbf{r}, z)=(m / 2)\left(\omega_{\perp}^{2} \mathbf{r}^{2}+\omega_{z}^{2} z^{2}\right)$. Here and henceforth $\mathbf{r}=(x, y)$. For simplicity, we will focus on a pancake-shaped trapping potential with $\omega_{z}^{2} /\left(\omega_{\perp}^{2}-\Omega^{2}\right) \gg 1$ such that the condensate can be regarded as quasi-two-dimensional whose motion along the $z$-axis is frozen to the zero-point motion of the axial harmonic oscillator.

\section{Fluctuations of the vortex lattice}

\subsection{Small fluctuations: large v and low temperatures}

At high $v$ and low temperatures $\left(T \ll T_{c}\right)$, quantum and thermal fluctuations are insignificant and the vortex lines organize into $2 \mathrm{D}$ solid which possesses the structure of a triangular array of lines parallel to axis of rotation with density $n_{v}$ (see Fig. 1). If we let $\bar{\rho}_{c}$ and $\bar{\rho}_{n}$ define the average condensate and non-condensate densities respectively, then this regime may be characterized by $\bar{\rho}_{n} \ll \bar{\rho}_{c}$. In other words, the Bose fluid in this regime is found to be almost completely described by the condensate wavefunction $\Phi(\mathbf{r})$ which is normalized as $\int|\Phi(\mathbf{r})|^{2} d \mathbf{r}=N_{c}$ where $N_{c}$ is the number of atoms in the condensate, and obeys the Gross-Pitaevskii equation [13] (taking $\hbar=m=1$ ):

$$
i \frac{\partial \Phi}{\partial t}=\left[h_{\perp}(\Omega)+g_{2 D}|\Phi(\mathbf{r})|^{2}\right] \Phi,
$$

where $g_{2 D}$ is the effective 2D strength of the contact interaction, and the linear operator $h_{\perp}$, written in the frame co-rotating with the trapping potential, takes the explicitly gauge invariant form:

$$
h_{\perp}(\Omega)=\frac{\left(-i \nabla_{\perp}-\boldsymbol{\Omega} \times \mathbf{r}\right)^{2}}{2}+\frac{1}{2}\left(\omega_{\perp}^{2}-\Omega^{2}\right) \mathbf{r}^{2} .
$$

In analogy with the Hamiltonian describing a charged particle in a uniform magnetic field [10], we identify a vector potential associated with rotation as $\mathbf{A}_{g}=\boldsymbol{\Omega} \times \mathbf{r}$. 
Let $\Phi_{0}(\mathbf{r}, t)=\Phi(\mathbf{r}) e^{-i \mu t}$ be a solution of Eq. (1), with $\mu$ being the chemical potential. Then $\Phi(\mathbf{r})$ satisfies the time-independent Gross-Pitaevskii equation:

$$
\left[h_{\perp}(\Omega)+g_{2 D}|\Phi(\mathbf{r})|^{2}\right] \Phi=\mu \Phi .
$$

Linearizing Eq. (1) around this solution by including small fluctuations of the form:

$$
\delta \Phi(\mathbf{r}, t)=e^{-i \mu t}\left[u(\mathbf{r}) e^{-i \omega t}-v^{*}(\mathbf{r}) e^{i \omega t}\right],
$$

we have the linearly coupled eigenvalue equation for $u(\mathbf{r})$ and $v(\mathbf{r})$ :

$$
\left(\begin{array}{ll}
h_{\perp}(\Omega)+2 g_{2 D}|\Phi(\mathbf{r})|^{2}-\mu & -g_{2 D}[\Phi(\mathbf{r})]^{2} \\
-g_{2 D}\left[\Phi^{*}(\mathbf{r})\right]^{2} & h_{\perp}(-\Omega)+2 g_{2 D}|\Phi(\mathbf{r})|^{2}-\mu
\end{array}\right)\left(\begin{array}{l}
u_{n} \\
v_{n}
\end{array}\right)=\omega_{n}\left(\begin{array}{l}
u_{n} \\
-v_{n}
\end{array}\right) .
$$

If we identify the zero-energy mode, i.e., $\omega_{0}=0$ and $u_{0}=v_{0}^{*}$ with $\Phi$, then Eq. (4) yields Eq. (3). So Eq. (3) simply represents the nonlinear portion of Eq. (4) which describes a set of coupled oscillators each of which interact only with the zero-energy mode. The normalization of the other eigenvectors $\left\{u_{n}(\mathbf{r}), v_{n}(\mathbf{r})\right\}_{n=1}^{n_{\max }}$ is given by:

$$
\int\left[u_{n}^{*}(\mathbf{r}) u_{m}(\mathbf{r})-v_{n}^{*}(\mathbf{r}) v_{m}(\mathbf{r})\right] d \mathbf{r}=\delta_{m n}
$$

where $\delta_{m n}$ is the Kronecker delta. This non-positive definite structure of Eq. (5) follows directly from the quantum statistics of the bosons [13]. In this formalism the total particle density $\rho(\mathbf{r})$ is given by:

$$
\rho(\mathbf{r})=|\Phi(\mathbf{r})|^{2}+\underbrace{\sum_{n=1}^{n_{\max }}\left[\frac{\left|u_{n}(\mathbf{r})\right|^{2}+\left|v_{n}(\mathbf{r})\right|^{2}}{\exp \left(\omega_{n} / T\right)-1}+\left|v_{n}(\mathbf{r})\right|^{2}\right]}_{\rho^{\prime}},
$$

where we have explicitly separated out the contribution of non-condensate $\rho^{\prime}$. In the above definition of $\rho^{\prime}$, we have cut off the summation at $n_{\max }$, or equivalently, we have introduced an energy cutoff $\omega_{c}=\omega_{n_{\max }}$. The sum in Eq. (6) converges very slowly and neglecting the modes above the cutoff may introduce unwanted errors in the calculation, thus making thermodynamic quantities rather sensitive to the cutoff. To improve upon this approximation, one can treat the above-cutoff modes semiclassically in the spirit of local density approximation [15], in which the normal modes take plane wave forms:

$$
u_{n}(\mathbf{r}) \rightarrow u(\mathbf{k}, \mathbf{r}) e^{i \mathbf{k} \cdot \mathbf{r}}, \quad v_{n}(\mathbf{r}) \rightarrow v(\mathbf{k}, \mathbf{r}) e^{i \mathbf{k} \cdot \mathbf{r}},
$$

with the normalization condition $|u(\mathbf{k}, \mathbf{r})|^{2}-|v(\mathbf{k}, \mathbf{r})|^{2}=1$. By inserting (7) into (4), one can readily derive the algebraic equations satisfied by $u(\mathbf{k}, \mathbf{r})$ and $v(\mathbf{k}, \mathbf{r})$ whose solution is rather straightforward [15].

\subsection{Large fluctuations: small $v$ and high temperatures}

The small-fluctuation assumption underlying Eqs. (3) and (4) implies that $\rho^{\prime} \ll \rho$. However, as the importance of $\rho^{\prime}$ grows with $T$ and/or a decrease in $v$, we have to make the replacement $g_{2 D}|\Phi(\mathbf{r})|^{2} \rightarrow g_{2 D} \rho(\mathbf{r})$ in Eq. (4) which makes it now a nonlinear equation. The same procedure is taken for the off-diagonal terms in Eq. (4) with the replacement $g_{2 D} \Phi(\mathbf{r})^{2} \rightarrow g_{2 D} \Delta(\mathbf{r})$, where

$$
\Delta(\mathbf{r})=-[\Phi(\mathbf{r})]^{2}-\underbrace{\sum_{n=1}^{n_{\max }}\left[\frac{2 u_{n}(\mathbf{r}) v_{n}^{*}(\mathbf{r})}{\exp \left(\omega_{n} / T\right)-1}+u_{n}(\mathbf{r}) v_{n}^{*}(\mathbf{r})\right]}_{\Delta^{\prime}} .
$$

Similar to the discussion in the previous section, we can include the above-cutoff contribution in $\Delta^{\prime}$ using the local density approximation. The quantity $\Delta^{\prime}$ defined above is the so-called anomalous average. It is well known that there is an ultraviolet divergence associated with this term when a bare contact interaction is adopted. Hence a proper renormalization procedure is required, the details of which can be found in Ref. [11]. A significant off-diagonal element also changes the Gross-Pitaevskii equation whose stationary form now becomes [13]:

$$
\left[h_{\perp}(\Omega)+g_{2 D} \rho(\mathbf{r})\right] \Phi+\Delta^{\prime} \Phi^{*}=\mu \Phi .
$$


Thus, in the most general context, the discretized problem that we are trying to solve may be clearly written down as a coupled nonlinear eigenvalue equation of the form:

$$
\left[\begin{array}{cc}
A & B \\
B^{*} & A^{*}
\end{array}\right]\left[\begin{array}{l}
u_{n} \\
v_{n}
\end{array}\right]=\omega_{n} \underbrace{\left[\begin{array}{cc}
I & 0 \\
0 & -I
\end{array}\right]}_{\eta}\left[\begin{array}{l}
u_{n} \\
v_{n}
\end{array}\right],
$$

where $I$ stands for the unit matrix, $A$ is hermitian $\left(A=A^{\dagger}\right)$ and $B$ is symmetric $\left(B=B^{T}\right)$ whose meanings can easily be inferred from the corresponding operators in Eq. (4). Quadratic Hamiltonians such as Eq. (10) occur frequently in physics because they are often the end result when a mean-field approximation is applied to a more complicated Hamiltonian and constitute the exact description for a fictitious system in which only two-point correlations exist (see Ref. [3] for a thorough discussion).

In the special case of a rotating BEC, the emergence of a vortex lattice introduces a class of low-energy excitations which are the Achilles heel of numerical calculations. These excitations are transverse waves known as Tkachenko waves [5,1] and they are governed by a very weak shear modulus which approaches zero when $\Omega \rightarrow \omega_{\perp}$. Consider a scenario in which $\Omega$ is continuously increased (see Fig. 1). The Tkachenko modes increase in number and, as a result of the decreased shear modulus, their frequencies become increasingly clustered around $\omega_{0}=0$. On the other hand we see from Fig. 1 that we need an increased domain with higher mesh densities on the boundary to accommodate the increasing velocity there. Eventually finite computational resources will be outstripped and the question then becomes what, if any, physical properties can be extracted beyond the regime where the Tkachenko frequencies cannot be resolved and the states are mixed by perturbations arising from discretization.

There is, of course, a physical distinction between thermal fluctuations and quantum fluctuations. This issue is closely related to an important problem of theoretical physics: the connection between a thermal and quantum phase transition or for that matter a partition function and a path integral. We defer a discussion of this to a future presentation of our physical results and for now, note that in both Eqs. (6) and (8), $\rho^{\prime}$ and $\Delta^{\prime}$ diverge with decreasing $v$ at constant $T$ or increasing $T$ at constant $v$. In both cases we are ultimately left with a problem of self-consistently solving a very large matrix problem with the structure of Eq. (10) and possessing low-lying eigenvalues clustered arbitrarily close to $\omega_{0}=0$.

\section{Solving the eigen problem}

In this section we turn our attention to solving Eq. (10). Our scheme anticipates that there are many instances of this solution in which the condensate wavefunction $\Phi(\mathbf{r})$ must be given special treatment. Here and henceforth, the replacements $g_{2 D}|\Phi(\mathbf{r})|^{2} \rightarrow g_{2 D} \rho(\mathbf{r})$ and $g_{2 D}[\Phi(\mathbf{r})]^{2} \rightarrow g_{2 D} \Delta(\mathbf{r})$ are implicit in any reference to Eq. (4). We take a top down approach and first generally describe the numerical scheme in its entirety before backtracking and introducing some details into each of the steps involved. Let $\left(\Phi_{j}^{i n}, \rho^{\prime i n}, \Delta_{j}^{\prime i n}\right)$ represent $\left(\Phi, \rho^{\prime}, \Delta^{\prime}\right)$ at the beginning of the $j^{\text {th }}$ iteration. For the $j^{\text {th }}$ iteration, the following steps are carried out:

(1) Solve Eq. (9) for $\Phi_{j}^{\text {out }}$ by Newton's method.

(2) With input as $\left(\Phi_{j}^{\text {out }}, \rho_{j}^{\prime i n}, \Delta_{j}^{\prime i n}\right)$ solve Eq. (4) for $\left\{u_{n}(\mathbf{r}), v_{n}(\mathbf{r})\right\}_{n=1}^{n_{\max }}$.

(3) From Eqs. (6) and (8), calculate $\rho_{j}^{\prime \text { out }} \underset{j}{\text { and } \Delta^{\prime \text { out }}}{ }_{j}$.

(4) Obtain the $\rho_{j+1}^{\prime}$ and $\Delta_{j+1}^{\prime}$ which will serve as input for the next iteration using modified Broydens mixing scheme with $\alpha \in(0,1)[12]$ :

$$
\begin{aligned}
& \rho_{j+1}^{\prime \text { in }}=F_{\rho}^{j}\left(\alpha, \rho_{j}^{\prime \text { in }}, \rho_{j}^{\prime \text { out }}\right) \\
& \Delta_{j+1}^{\text {in }}=F_{\Delta}^{j}\left(\alpha, \Delta_{j}^{\prime \text { in }}, \Delta_{j}^{\prime \text { out }}\right) .
\end{aligned}
$$




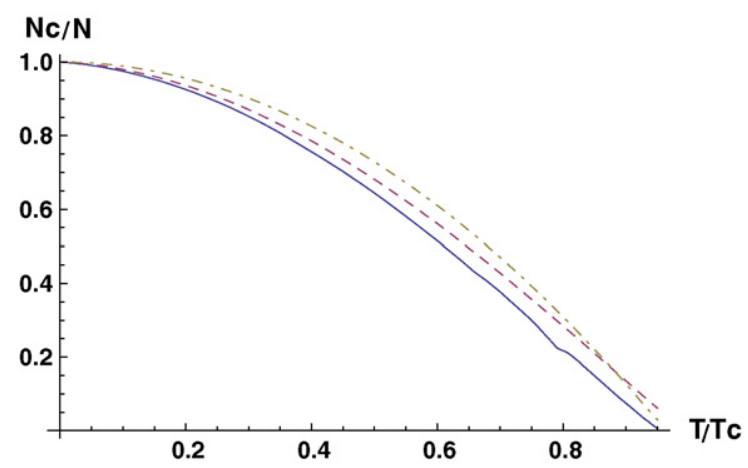

Fig. 2. Condensate fraction as a function of temperature obtained from 3 different physical systems. Briefly these are: Dotted-dashed curve-A stationary ideal gas. Here we use a direct numerical sum of the occupation numbers of a 2-Dimensional harmonic oscillator; Dashed curve-A stationary interacting Bose gas obtained by solving Eq. (10); Unbroken curve-A slowly rotating condensate which has one vortex at $T=0$, also obtained from a solution of Eq. (10). Here $T_{c}$ refers to the transition temperature for an ideal bosonic gas.

Here $F_{\rho}$ and $F_{\Delta}$ are symbols for the mixing algorithm outlined in [12]. Set $\Phi_{j}^{\text {out }}$ to $\Phi_{j+1}^{\text {in }}$ and repeat from step (1) until $\left|\mu_{j}-\mu_{j-1}\right|<\delta$, where $\delta$ is a chosen tolerance related to the expected accuracy of the discretization.

A key element in the scheme outlined above, is the special treatment which we give to the condensate. This underlies a special difficulty of dealing with bosons: away from a phase transition, the lowest mode is as important, if not more, than the combined weight of all the other modes. Thus, in contrast to electronic structure calculations, one needs, in the case of a condensate, the extra step (1) above.

To solve Eq. (9) by Newton's method we enforce the renormalization condition of the condensate wavefunction by adding a term $(\beta / 2)\left(\int|\Phi|^{2} d \mathbf{r}-N_{c}\right)^{2}$ where $\beta \gg 1$, to convert the problem to one of the unconstrained minimization. We then solve the new modified equation using Newton's method with a quadratic line-search or a trust-region method [7].

We work completely in the context of PETSc [2] and SLEPc [9] libraries which are built for parallel computation and within which all the necessary matrix-vector manipulations are easily performed. By taking into account that $\eta^{2}=I$, multiplying through Eq. (10) yields an unsymmetric system with a positive inner product. However to avoid writing our own eigensolver which will take advantage of this symmetry, we solve the unsymmetric form with freely available libraries [9]. For large lattices, Eq. (4) is very sensitive and even in the linear regime, yields some complex eigenvalues which could only mean that $\Phi$ is not properly converged or that the discretization or the unsymmetric solution is slightly perturbing the solution.

\section{Illustrative results and practical considerations}

\subsection{Some computational results}

Before we delve into computational details, we proceed to emphasize a few important points by showcasing some simple results. In Fig. 2 we compare the condensate fraction as a function of temperature for 3 different situations: an ideal non-rotating gas, an interacting non-rotating gas and a slowly rotating interacting gas which sustains one vortex at $T=0$. From physical considerations, the presence of interactions and vortices are expected to reduce the condensate fraction from that of an ideal gas at the same temperature. As a consequence, the temperature at which the condensate vanishes is also expected to be lower when vortices and interactions increase. We observe from the Fig. 2 that our results are not strictly consistent with this expectation when $T \rightarrow T_{c}$. This occurs because the bogoliubov amplitudes $u(\mathbf{k}, \mathbf{r})$ and $v(\mathbf{k}, \mathbf{r})$ (see Section 3.1) converge very slowly as $|r| \rightarrow \infty$ and for practical purposes, since it is not our purpose to accurately determine the transition temperature, we truncate the sum prematurely by performing our calculation in a relatively small domain. This omission underestimates the value of $\rho^{\prime}$.

Another important issue that should be emphasized is that as the calculation proceeds towards a stationary state, the number of vortices and the symmetry of their arrangement can change. In our example, as the temperature is raised, a 

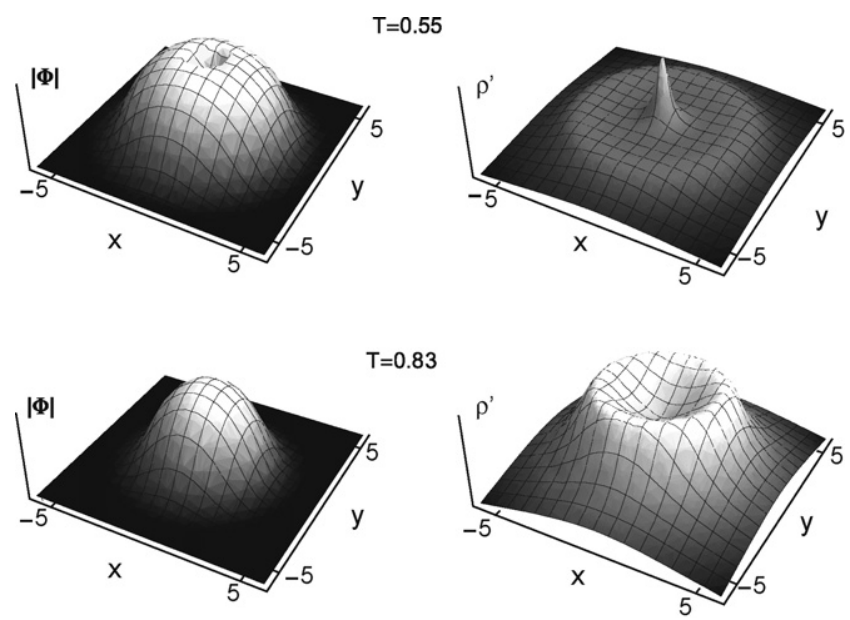

Fig. 3. Density profiles for the condensate wavefunction $\Phi$ and normal fluid $\rho^{\prime}$ at $T=0.55 T_{c}$ and $T=0.83 T_{c}$ for a slowly rotating condensate (see unbroken curve in Fig. 2). As the temperature is increased, the vortex is ejected from the condensate.

system with one vortex shown in the upper panel of Fig. 3 evolves into the system shown in the lower panel by ejecting the vortex.

Lastly, the specter of a ground state with a different symmetry than the initial state also emphasizes the need for a more sophisticated convergence algorithm than simple mixing which has been found to be inadequate or cumbersome in such situations [12]. In our calculations, we find that modified Broydens required dozens of iterations to converge when such symmetry changes were occurring.

\subsection{Operators and functions in a finite element space}

In our calculations, we make exclusive use of linear triangular elements. On each triangle, any function $\Phi^{h}(\mathbf{r})$ defined in the finite element space is approximated by a plane: $\Phi_{i}^{h}(\mathbf{r})=a_{i}[1] x+a_{i}[2] y+a_{i}[3]$, where the the subscript $i$ labels the index of element. We take $h$ to represent the radius of the circum-circle of the triangle. We ensure continuity of $\Phi^{h}$ by choosing its values at the 3 vertices $\left\{\phi_{i}^{h}[1], \phi_{i}^{h}[2], \phi_{i}^{h}[3]\right\}$ as the 3 degrees of freedom per element. Let $\vec{a}_{i}=\left(a_{i}[1], a_{i}[2], a_{i}[3]\right)$ and $\vec{v}_{i}=\left(\Phi_{i}[1], \Phi_{i}[2], \Phi_{i}[3]\right)$ are related by the matrix $T_{i}$ through: $\vec{a}_{i}=T_{i} \vec{v}_{i}$. For a specific example, let us examine how the expectation value of a hermitian operator $\hat{p}$ yields expression in the finite element space:

$$
\begin{aligned}
& \langle\hat{p}\rangle=\sum_{i} \int_{\Delta} \phi_{i}^{h *} \hat{p} \phi_{i}^{h} d \mathbf{r} \\
& =\sum_{i}\left[\begin{array}{lll}
a_{i}[1] & a_{i}[2] & a_{i}[3]
\end{array}\right] \bullet \int_{\Delta}\left[\begin{array}{lll}
p_{11}(x, y) & p_{12}(x, y) & p_{13}(x, y) \\
p_{21}(x, y) & p_{22}(x, y) & p_{23}(x, y) \\
p_{31}(x, y) & p_{32}(x, y) & p_{33}(x, y)
\end{array}\right] d \mathbf{r} \bullet\left[\begin{array}{l}
a_{i}[1] \\
a_{i}[2] \\
a_{i}[3]
\end{array}\right] \\
& =\sum_{i} \vec{a}_{i}^{T} \cdot \int_{\Delta} P(x, y) d \mathbf{r} \cdot \vec{a}_{i} \\
& =\sum_{i} \vec{v}_{i}^{T} \cdot\left(T_{i}\right)^{T} \cdot \int_{\Delta} P(x, y) d \mathbf{r} \cdot T_{i} \cdot \vec{v}_{i} \\
& =\sum_{i} \vec{v}_{i}^{T} \cdot P_{i}^{\prime} \cdot \vec{v}_{i},
\end{aligned}
$$

where the sum $\sum_{i}$ is taken over all elements in the domain and the integral $\int_{\Delta} \cdots d \mathbf{r}$ is taken over each triangular element. In Eq. (12) we see that the elements of $P_{i}$ are obtained through quadrature of the triangular area supporting 

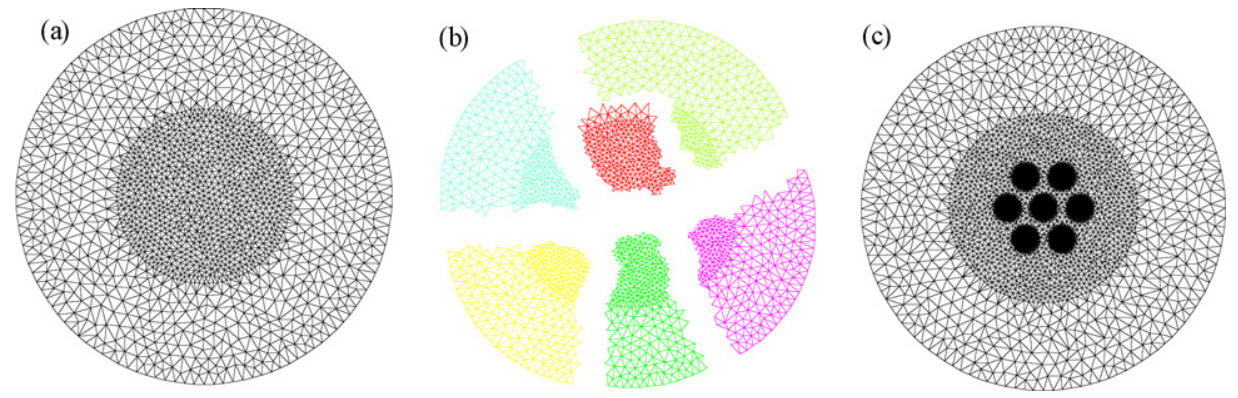

Fig. 4. In a) we show a typical mesh employed in our calculations. A finely meshed condensate region is surrounded by a coarser mesh for the boundary where thermal particles reside at $T \gg 0$ (see Fig. 3). b) A partition of the mesh in a) for parallel compuations using METIS. c) A more complicated mesh pattern anticipating a groundsate lattice such as in Fig. $1 \mathrm{~b}$ with a very fine mesh for the vortex core regions.

the element. For this task we find the Wandzura quadrature rules [17] very suitable. Although we have presented a discussion on linear elements, our methods are easily extensible to higher order elements.

\subsection{Matrix assembly}

It is now clear how to assemble the matrix $P$. On each element $i, P_{i}$ corresponds to a submatrix of $P$ and $\left(\phi_{i}^{h}[1], \phi_{i}^{h}[2], \phi_{i}^{h}[3]\right)$ maps onto $\left(\Phi^{h}[k], \Phi^{h}[l], \Phi^{h}[m]\right)$ where $(k, l, m)$ are the global indices of the nodes $(1,2,3)$ of triangle $i$ in a numbering scheme typically chosen to facilitate matrix computations. For details on matrix assembly we refer to Ref. [16]. We make a quick point on the origin of the sparsity of the problem. The $n_{b}$ neighboring points around each node roughly define a $n_{b}$-sided polygon. If the smallest angle that the $k^{\text {th }}$ node subtends in a triangle is $\alpha$, then it can have up to $2 \pi / \alpha$ neighbors which suggests why the finite element matrices are very sparse. If $\alpha$ is the smallest angle in mesh then no node can have more than $2 \pi / \alpha$ neighbors.

\subsection{Error analysis}

The error in our finite element approximation on each triangle is given by:

$$
\int_{\Delta}\left|\Phi(\mathbf{r})-\phi_{i}^{h}(\mathbf{r})\right| \leq C|\Phi|_{k} h^{k}
$$

where $C$ is a constant which depends upon the structure of the element, $(k-1)$ is the order of the equation (in our case $k=2)$ and $|\Phi|_{k}=\sum_{\beta_{1}+\beta_{2}=k} \int\left|\left(\partial^{k} \Phi\right) /\left(\partial x^{\beta_{1}} \partial y^{\beta_{2}}\right)\right|^{2}$. One way to understand Eq. (13) is to see the finite element approximation as a local Taylor expansion with the order of the expansion corresponding to that of the approximating polynomial. By inference it should be clear from Eq. (13) why such a fine mesh is usually required to model a vortex lattice. By definition $\left|\mathbf{v}_{c}\right| \propto 1 /|\mathbf{r}|$ in the neighborhood of a vortex which makes $|\Phi|_{k} h^{k}$ very big. Consequently one requires very small triangles even for an approximate description. In addition the coarse grained velocity field $(\boldsymbol{\Omega} \times \mathbf{r})$ can get very high close to the boundary of a large condensate and this problem is superimposed on the singularity of the core. Estimating the error is especially important in gauging the suitability of the mesh to a predetermined spectral accuracy. As we mentioned above, the vortex lattice gives rise to several low-lying excitations clustered around the origin which must be resolved in any reliable spectroscopic calculation of Eq. (4).

\subsection{Choosing a mesh}

In the mesh depicted in Fig. 4a, which is generated using the mesh generator TRIANGLE (http://www.cs.cmu.edu/ quake/triangle.html), we have broken the domain into two regions, an inner region with very small triangles supporting the condensate and an outer region with larger triangles which supports the normal fluid when $T>0$. One key difficulty of working with an unstructured mesh in a parallel computation is the need to partition the nodes among the processors to reduce communication costs. In Fig. 4b, we display a partition of the mesh from Fig. 4a into 6 parts using the the METIS graph partitioning library (http://glaros.dtc.umn.edu/gkhome/views/metis) 
which meets our needs quite well. During our initial studies there was the temptation for more elaborate meshing schemes. For instance, for a particular lattice structure as shown in Fig. 1b, we may refine the vortex cores such as shown in Fig. 4c. However we quickly abandoned the idea for one main reason: as fluctuations grow, the vortex lattice structure and the vortex positions can change significantly. This development annuls any meshing scheme which anticipates that the vortex positions will remain fixed. We also find even for the case where fluctuations are small that the trade-off between the increased complexity of the program and the savings in reduction of computational costs is not very attractive. At the time of writing most of our calculations have relied on a two-region meshing structure shown in Fig. 4a.

\section{Conclusion and outlook}

We have discussed a numerical approach to studying a vortex lattice in a rapidly rotating BEC in the presence of high quantum or thermal fluctuations. This physical system presents a very large unsymmetric eigen problem with eigenvalues clustered arbitrarily close together and the solution of which requires state-of-the-art numerical algorithms and computational techniques. The outstanding physical problem is to determine if one can extract physically meaningful properties of the two-point correlations in a regime when the underlying discretization cannot resolve all the closely spaced excitations. On the other hand, the outstanding computational problem is to develop a specialized method for solving the problem whilst taking advantage of its symmetric structure.

The method we outlined here also applies to superfluid Fermi gases. Under the mean-field framework, the properties of the Fermi gas are described the the Bogoliubov-de-Gennes equation [6] which is the fermionic counterpart of Eq. (4). The procedure of solving the equation follows the same steps described in Section 4 with step (1) eliminated since here we do not have a condensate.

We are grateful to R.N. Barnett for providing a subprogram for the modified Broydens method [12]. All the computations presented in this paper were done at the Center for Computational Materials science, Georgia Institute of Technology.

We gratefully acknowledge the financial support from the National Science Foundation and the Robert A. Welch Foundation (Grant No. C-1669).

\section{References}

[1] L.O. Baksmaty, S.J. Woo, S. Choi, N.P. Bigelow, Tkachenko waves in rapidly rotating Bose-Einstein condensates, Phys. Rev. Lett. 92 (2004) 160405.

[2] S. Balay, K. Buschelman, W.D. Gropp, D. Kaushik, M.G. Knepley, L.C. McInnes, B.F. Smith, H. Zhang, http://www.mcs.anl.gov/petsc, PETSc parallel libraries, 2001.

[3] J.-P. Blaizot, G. Ripka, Quantum Theory of Finite Systems, The MIT Press, London, England, 1985.

[4] M.A. Cazalilla, Surface modes of ultracold atomic clouds with a very large number of vortices, Phys. Rev. A 67 (2003) 063613.

[5] I. Coddington, P. Engels, V. Schweikhard, E.A. Cornell, Observation of Tkachenko oscillations in rapidly rotating Bose-Einstein condensates, Phys. Rev. Lett. 91 (2003) 100402.

[6] P. de Gennes, Superconductivity of Metals and Alloys, Perseus Books, 1999.

[7] J.E. Dennis Jr., R.B. Schnabel, Numerical Methods for Unconstrained Optimization and Nonlinear Equations, Prentice-Hall, Inc., Englewood Cliffs, NJ, 1983.

[8] R.J. Donnelly, Quantized Vortices in Helium II, Cambridge University Press, Cambridge, 1991.

[9] V. Hernandez, J.E. Roman, V. Vidal, SLEPc: a scalable and flexible toolkit for the solution of eigenvalue problems, ACM Trans. Math. Softw. $31(2005) 351$.

[10] T.-L. Ho, Bose-Einstein condensates with large number of vortices, Phys. Rev. Lett. 87 (2001) 060403.

[11] D.A. Hutchinson, R.J. Dodd, K. Burnett, Gapless finite-T theory of collective modes of a trapped gas, Phys. Rev. Lett. 81 (1998) 2198 ; N.P. Proukakis, S.A. Morgan, S. Choi, K. Burnett, Comparison of gapless mean-field theories for trapped Bose-Einstein condensates, Phys. Rev. A 58 (1998) 2435.

[12] D.D. Johnson, Modified Broyden's method for accelerating convergence in self-consistent calculations, Phys. Rev. B 38 (1988) 12807.

[13] A.J. Leggett, Bose-Einstein condensation in the alkali gases: some fundamental concepts, Rev. Mod. Phys. 73 (2001) 307.

[14] L.M. Pismen, Vortices in Nonlinear Fields, Clarendon Press, Oxford, 1999.

[15] J. Reidl, A. Csordás, R. Graham, P. Szépfalusy, Finite-temperature excitations of Bose gases in anisotropic traps, Phys. Rev. A 59 (1999) 3816.

[16] G. Strang, G.G. Fix, An Analysis of the Finite Element Method, Wellesly-Cambridge Press, 1973.

[17] S. Wandzura, H. Xiao, Symmetric quadrature rules on a triangle, Comput. Math. Appl. 45 (2003) 1829. 